\title{
Standounkt
}

\section{Homo Faber zwischen Hoffnung und Hybris}

\begin{abstract}
Klimaprobleme mit technischen Eingriffen in das Erdsystem lösen - diesen Ansatz haben diverse Geoengineering-Technologien gemeinsam. Weil Emissionssenkung und Klimaanpassung bisher nicht ausreichend umgesetzt wurden, könnte Geoengineering eine letzte Hoffnung sein. Oder ein weiterer Schritt in Richtung Hybris und menschliche Selbstzerstörung. Von Christian Uhle
\end{abstract}

W ir leben technisch, der Mensch als Beherrscher der Natur, der Mensch als Ingenieur, und wer dagegenredet, der soll auch keine Brücke benutzen, die nicht die Natur gebaut hat", meint Walter Faber in dem bekannten Roman von Max Frisch.

Faber irrt sich. Jede Technologie wirft eigene Fragen auf über das Verhältnis des Homo Sapiens zu sich und seiner Umwelt. Gegenwärtig besteht besonders dringender Anlass, solche Fragen im gesellschaftlichen Diskurs zu verhandeln. Denn in Fachkreisen wird die Möglichkeit diskutiert, dem fortschreitenden Klimawandel durch massive Eingriffe in das Erdsystem zu begegnen.

\section{Bequem und verlockend}

Unter dem Begriff Geoengineering werden Vorschläge zusammengefasst, die entweder darauf abzielen, die Sonneneinstrahlung zu verringern oder der Atmosphäre Kohlenstoffdioxid zu entziehen. Beispielsweise wird diskutiert Schwefelpartikel in die Atmosphäre einzubringen, Verwitterungsprozesse zu beschleunigen, Ozeane zu düngen oder durch künstliche Bäume Kohlenstoff aus der Luft zu filtern. Gegenüber jedem dieser Ansätze wurden Bedenken geäußert. Zum Beispiel gehen Klimaforscher/innen davon aus, dass ein weltumspannender Schirm aus Schwefelpartikeln verheerende Auswirkungen auf Niederschlagsmuster hätte. Demgegenüber sehen Befürworter/innen in diesen Technologien einen letzten Ausweg aus der drohenden Klimakatastrophe oder sogar eine vielversprechende Möglichkeit zur Gestaltung von Klima und Erdsystem. Das „Anthropozän“ würde zum Prinzip erhoben.

Auf den ersten Blick klingt es bequem und verlockend, die Umwelt an menschliche Bedürfnisse anzupassen, statt umgekehrt. Beim Brückenbau hat sich diese Denkweise schließlich auch bewährt. Anders sieht es jedoch bei Eingriffen in komplexe natürliche Systeme aus. Beispielsweise werden meist mehr Probleme verursacht als gelöst, wenn man versucht Ökosysteme durch die Einführung räuberischer Spezies zur Schädlingsbekämpfung zu gestalten. Die Folgen solcher Eingriffe sind nicht zuverlässig modellier- und vorhersehbar. Grund ist auch, dass Wechselwirkungen in komplexen und nichtlinearen Systemen so vielschichtig sind, dass kleine Änderungen einzelner Parameter große Auswirkungen auf das Gesamtsystem haben können. Schon aufgrund solcher „Butterfly-Effekte" liegt der Verdacht auf Hybris nahe, wenn wir nun Strahlungshaushalt und Klima der gesamten Erde kontrollieren wollen. Hier greifen wir in höchst komplexe, chaotische Systeme ein und riskieren Nebeneffekte auf globaler Ebene. Zweifelhaft wäre dieses Vorgehen auch, weil uns technikgestützte Eingriffe in das Erdsystem und die Ignoranz planetarer Grenzen in die gegenwärtige, gefährliche Lage gebracht haben. Es ist unwahrscheinlich, dieses Problem durch immer neue technische Eingriffe lösen zu können. In den seltensten Fällen kön- nen Probleme mit derselben Denkweise gelöst werden, durch die sie entstanden sind, warnte Albert Einstein.

Aber selbst wenn es möglich wäre, das Klimasystem in einem solchen Sinne zu kontrollieren, wäre es überhaupt wünschenswert in einer gänzlich technisierten Welt zu leben, in der auch das Klima zum menschlichen Artefakt wird? Oder wäre eine demütigere Haltung und der Versuch, das eigene Handeln in Einklang mit den planetaren Grenzen zu bringen, einem guten Leben förderlicher?

Diese Fragen gehen jede/ $\mathrm{n}$ an, und können nur in einem breiten Diskurs beantwortet werden. Es ist falsch, dass Geoengineering derzeit fast nur in Fachkreisen verhandelt wird. Folgen von Geoengineering betreffen potenziell die ganze Menschheit. Entsprechend sollten Entscheidungen hierüber demokratischer und öffentlichkeitswirksamer erfolgen als gegenwärtig. Und nur eine aufgeklärte Öffentlichkeit kann verhindern, dass aufgrund möglicher Geoengineering-Technologien die Notwendigkeit zur Emissionssenkung infrage gestellt wird.

\section{Noch nicht umsetzbar}

Viele Ansätze von Geoengineering sind noch nicht umsetzungsfähig. Geforscht wird aber bereits intensiv. In einer differenzierten Debatte muss zwischen den verschiedenen Vorschlägen von Geoengineering unterschieden werden. Jeder Geoengineering-Ansatz ist mit eigenen Gefahren und ethischen Problemen verbunden. Diese Probleme dürfen durch die Verlockung einer einfachen Lösung nicht ausgeblendet werden. Auch wenn der Mensch als Homo Faber technisch lebt, sollte er gründlich überlegen, inwieweit er sich zum Beherrscher des Klimas und damit des gesamten Erdsystems aufspielt.

AUTOR + KONTAKT

Christian Uhle ist Philosoph und arbeitet als wissenschaftlicher Mitarbeiter am Institut für Ökologische Wirtschafsforschung (IÖW).

Institut für ökologische Wirtschaftsforschung (IÖW) $\mathrm{GmbH}$, gemeinnützig, Potsdamer Str. 105, 10785 Berlin. Tel.: +49 30 884594-39, E-Mail: christian.uhle@ioew.de and redistributing the material in any medium or format, provided the original work is properly cited, it is not used for commercial purposes and it is not remixed, transformed or built upon. The access to the digital version of this article is reserved to subscribers of ÖkologischesWirtschaften until two years after the date of publication; after two years it is available to all readers. 